\title{
Experimental studies of the structure of biological tissues through mechanical effects with a smart laparoscopic instrument
}

\author{
Veronika Ivanova ${ }^{1}$, Ditchko Batchvarov ${ }^{2,}{ }^{*}$, Zlatoliliya Ilcheva ${ }^{2}$, Ani Boneva ${ }^{2}$, Svetozar Ilchev $^{2}$, \\ Alexander Alexandrov", and Rumen Andreev ${ }^{2}$ \\ ${ }^{1}$ Institute of Robotics - Bulgarian Academy of Sciences, Department of Robotized Executive Mechanisms and Intelligent Systems, \\ Acad. G. Bonchev str., bl. 1, Sofia 1113, Bulgaria, e-mail: iwanowa.w@abv.bg \\ ${ }^{2}$ Institute of Information and Communication Technologies - Bulgarian Academy of Sciences, Department of Communication \\ Systems and Services, Acad. G. Bonchev str., bl. 2, Sofia 1113, Bulgaria, e-mails: dichko1952@abv.bg, zlat@isdip.bas.bg, \\ a_boneva1964@abv.bg, svetozar@ilchev.net, rumen@isdip.bas.bg, \\ ${ }^{3}$ Institute of Information and Communication Technologies - Bulgarian Academy of Sciences, Department of Modelling and \\ Optimization, Acad. G. Bonchev str., bl. 2, room 302, Sofia 1113, Bulgaria, e-mail: akalexandrov@iit.bas.bg
}

\begin{abstract}
The article presents investigations in the area of the analysis of heterogeneous biological tissues using tools based on mechanical stimulations. Some known tissue mechanical models are presented along with one approach for specifying the tissue internal structure by measuring mechanical macro parameters of the research tools. The methodology of micro and macro stimulation is described and the construction of an experimental model of a smart laparoscopic tool for its realization is presented.
\end{abstract}

\section{Introduction}

Laparoscopic surgery rapidly evolves as minimally invasive surgery. Procedures are performed by long tin instruments inserted thought trocars. However, the lack of tactile sense is the cardinal disadvantage associated with laparoscopy, because it limits the surgeon's abilities to examine the internal organs of patients. For instance, when there are tumors, they tend to be harder than the surrounding tissue. It is not possible to indicate the presence, size and exact location of a tumor without tactile sense when the diagnostics is performed by laparoscopic procedure. Biological tissues are characterized as complex and heterogeneous structures. It is possible to identify diseases by investigating their local properties. The requirement for a rapid operative determination of the presence of an anomaly in the tissue structure needs the development of simple direct methods and analyses without the need for complex and slow processing of measurement results. There are many methods for diagnostics of gallbladder carcinoma: Blood tests, Ultrasound Computerized Tomography (CT) scan, Magnetic resonance imaging (MRI) Endoscopic retrograde cholangio pancreatography (ERCP) Biopsy, Laparoscopy, etc.

In the paper, we describe our efforts to solve the problem associated with inverse tissues characterizations in laparoscopic surgery. The method of analysis of the tissue structure proposed in the article has some advantages over the known direct ways for biological microstructure characterization. Among the more important of them are: $i$ ) the analysis is made on the basis of a micro-parameter $\tau$ (which determines the type of viscoelastic environment). This parameter is measured by means of macro-screening - Force and Time individually for each macro stimulus. There is no need to measure deformation and strain, which are often difficult to determine; ii) $\tau$ is the result from direct force measurements, which integrate the micro responses at every contact point for the given macro stimulus. In this context, the parameter filters the noise and reports the average relaxation time; iii) this method is useful for real-time diagnostics and force-feedback interacting in surgical robotics. It is possible to scan accurately the change of $\tau$ and locate precisely the areas where the parameter makes sharp changes in its value.

The remainder of the paper is organized as follows:

2) Biological tissues. Properties,

3) Mechanical models of biological tissues,

4) Micro and macro stimulating methods for the analyses of biological tissues,

5) A device for micro and macro stimulation,

6) An analysis of the output signal received from the device,

7) Discussion,

8) Conclusions.

\section{Biological tissues. Properties}

Biological tissues have specific characteristics in contrast to other non-biological engineering materials: $i$ ) tissues are adaptable and change their properties under external force effects; ii) tissue properties can also change as a result of ageing. Most biological tissues are composite materials (they include materials with

Corresponding author: dichko1952@abv.bg 
different properties) with heterogeneous and anisotropic structure - their properties can vary at different points. They change in length as a result of applied force. The tissues have a highly elastic behavior - they possess both the elasticity characteristic of solids and the viscosity characteristic of liquids. The main components defining the mechanical properties of tissues are fibers formed by two types of proteins - collagen and elastin. Collagen forms fibers by combining a set of filaments, which have a behavior similar to a set of connected springs in the fiber composition.

Biological tissues have both viscous and elastic properties - their deformation depends on the ratio of external forces and they lose some energy when returning to their shape. Therefore, collagen fibers exhibit both viscosity and elastic properties and they are responsible for the viscoelastic behavior of the tissues. Elastin also forms fibers that are more elastic than viscous.

It can be concluded that the collagen fibers define the viscoelastic properties of the tissues with a high modulus of elasticity, while the elasticity - the low modular elastic properties of the latter.

\section{Mechanical models of biological tissues}

Three mechanical models describe accurately biological tissues under external force effects. These methods are realized by an elastic body (a spring) and viscosity (different configurations of links). In these models, the following definitions are accepted:

$$
\varepsilon \text { - Strain, deformation, } \frac{\Delta L}{L} \text {, the ratio of change in }
$$

length of the object under an applied force to the length of the object before the applied force;

$$
\sigma \text { - Stress, } \frac{F}{S} \text {, a local parameter defining the force }
$$

per unit area at a given point of the object;

$\mathrm{s}$ - index, a variable quantity for an elastic element in the presented models;

d - index, a variable quantity for a viscous element in the presented models

Presented models show the local structure and tissue reactions represented depending on the values $\mathcal{E}$ and $\sigma$, at the real values of the parameters $E$ (elastic modulus for an elastic element) and $\eta$ (viscosity resistance in the viscous element of the model).

The real value of $\varepsilon$ and $\sigma$ cannot be measured at every tissue point, so it is important to determine their relationship.

In the Fig. 1, the Kelvin-Voight model is shown.

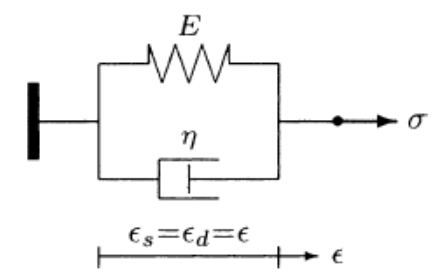

Fig. 1. Kelvin-Voight model.

From the Kelvin-Voight model, we get that the stress $\sigma$, strain $\varepsilon$ and their rates of change with respect to time $t$ are governed by equations of the form:

$$
\sigma=E \times \xi+\eta \frac{d \xi}{d t}
$$

where $E$ is the modulus of elasticity of the spring and $\eta$ is the viscous element.

The equation can be applied either to the shear stress or to the normal stress of a material. The KelvinVoigt model is effective for predicting creep; it is not good at describing the relaxation behavior after the stress load is removed.

The Maxwell Model is shown in Fig. 2.

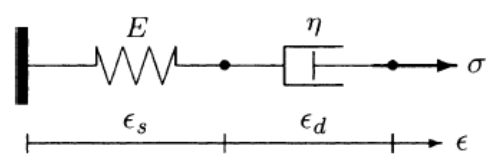

Fig. 2. Maxwell Model.

The Maxwell Model can be represented by the following equation:

$$
\eta \frac{d \sigma}{d t}+E \sigma=E \times \eta \frac{d \xi}{d t}
$$

where $E$ is the modulus of elasticity of the spring and $\eta$ is the ratio of strain of the viscous element.

The Maxwell model predicts a linear relationship between strain and time, which is most often not the case.

The Standard Linear Solid model is shown in Fig. 3.

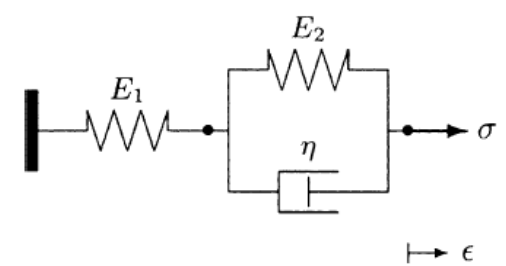

Fig. 3. Standard Linear Solid model.

The Standard Linear Solid model can be represented by the following equation:

$$
\left(E_{1}+E_{2}\right) \times \sigma+\eta \frac{d \sigma}{d t}=E_{1} \times E_{2} \times \xi+E_{1} \times \eta \frac{d \xi}{d t}
$$


Where $E_{1}$ and $E_{2}$ are the modulus of elasticity of two springs and $\eta$ is the ratio of strain of the viscous element.

These three models have been used to determine the local reactions of biological tissues.

Presumably, the force is applied at the given tissue point and the contact point is removed as a result of this. The reaction has an opposite direction to the force and is applied to the contact point where the force is applied. The first task in choosing an adequate working model is to determine the specific characteristics of the biological tissue - the elastic or viscous elastic groups. In general, there are relationships between $\varepsilon$ and $\sigma$ :

$$
\sigma=\sigma\left(\xi, \frac{d \xi}{d t}, \ldots, t\right)
$$

$\sigma=E \xi$ in the general case.

The tissues should be defined as belonging to an elastic or a viscoelastic group and the dependence $\mathcal{E}-\sigma$ is represented by the graphs in Fig. 4 and Fig. 5, respectively.

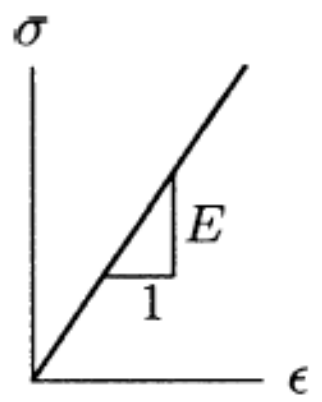

Fig.4. Elasic tissues.

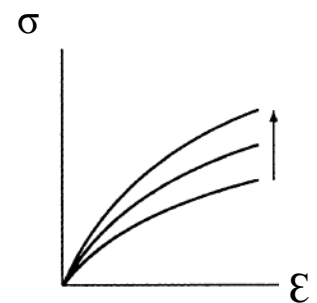

Fig. 5. Viscous elastic tissues.

The graph changes when $\varepsilon$ increases in a value.

Another feature is the presence of hysteresis when moving between two values of $\mathcal{E}$ in forward and reverse direction (loading and unloading, respectively). This is explained by the release of heat from the viscosity element, which reduces the potential energy accumulated in the elastic element during the first movement. In elastic tissues, hysteresis lacks and the trajectories of the two movements coincide.

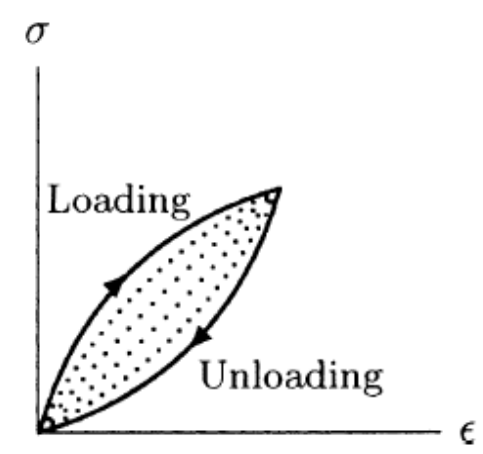

Fig. 6. Viscous elastic tissue. Hysteresis.

One possible way to determine the parameters of the chosen model in a viscous elastic environment is by performing a Time and Relaxation test including the formation of a value of $\xi=\xi_{0}$ at the start of the report. In subsequent moments, the tissue reaction is monitored by measuring $\sigma$.

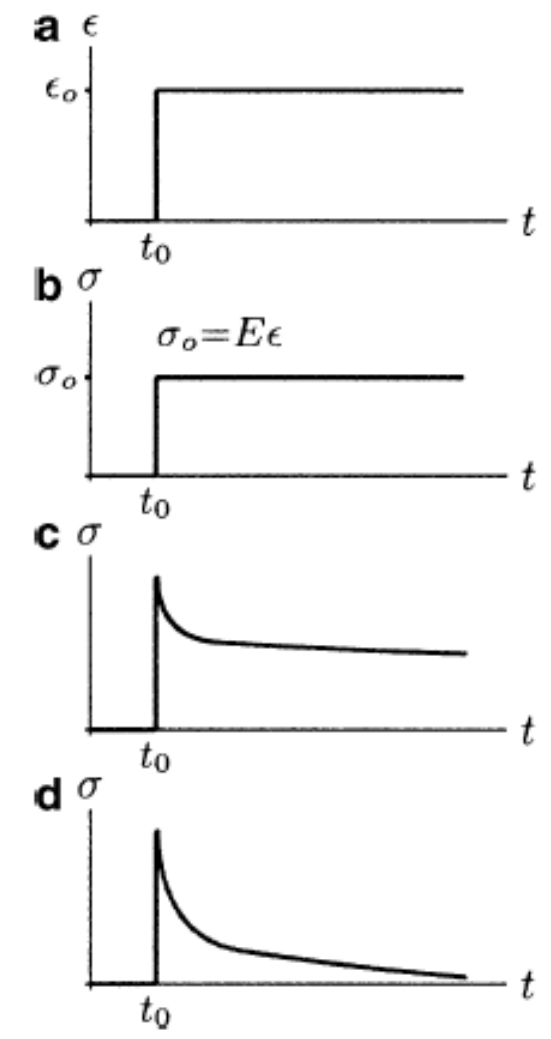

Fig. 7. A Time and Relaxation test.

In Fig $7 b$, the reaction of an elastic tissue is shown. In Fig. 7c and Fig. 7d, the reactions of viscous elastic tissues with different viscosity are shown.

The Maxwell model is appropriate for viscous elastic tissues therefore $\sigma$ can be evaluated as a function of time. We consider $t_{0}=0$ at the beginning of every Time and Relaxation test . 
At the beginning, when $\xi=\xi_{0}, \sigma_{0}=\xi_{0} \times E$ and $t=0, \sigma(t)$ is obtained from Equation 2:

$$
\sigma(t)=\xi_{0} \times E \times e^{-\frac{E}{\eta} t}
$$

This is a result from the requirement of the Time and Relaxation test ( $\xi=\xi_{0}, t=0$ ).

Therefore, the right part of the Equation (2) is always zero. $\frac{d \xi}{d t}=0$ and Equation (2) is reduced to:

$$
\eta \frac{d \sigma}{d t}+E \sigma=0
$$

After the replacement $\tau=\frac{\eta}{E}$, from equation (5) it follows that:

$$
\sigma(t)=\xi_{0} \times E \times e^{-\frac{t}{\tau}}
$$

Let $E_{r e l}(t)=\frac{\sigma(t)}{\xi_{0}}$ be a relative change of the Stress during the test. Then, equation (2) can be written as:

$$
\sigma(t)=\xi_{0} \times E_{r e l}(t)=\xi_{0} \times E \times e^{-\frac{t}{\tau}}
$$

Two parameters are involved - $\mathrm{E}$ and $\tau$. $\tau$ is the time for relaxation and it defines the time after which:

$$
\sigma(t)<\left(\varepsilon_{0} \times E\right) / e
$$

This parameter is fundamental for determining the properties of the viscous elastic tissue, as the relation $\frac{\eta}{E}$ allows the tissue to be classified and it remains unchanged under all stress deformations defined by $\xi \times E$.

Therefore, our target is to determine the microparameter $\tau$ by observing the real "macro quantities" associated with the response as a result of the deformation of the tissue, which can be controlled by a smart tool. The determination of $\tau$ is based on the actual "macro quantities" and will be described in the next section. In the process of a sequence of uniform stress deformations of the tissue and corresponding measurements of the $\tau$ values for each of them, the variation of this parameter can be interpreted as a mark for abnormal patterns in the tissue and a precondition for activating a particular operating mode of the robotic instrument.

\section{Micro and macro stimulating methods for analyses of biological tissues}

The macro and micro stimulation method includes a robot tool with incorporated force sensors. The force is measured by the sensors in the direction opposite to the instrument displacement. The instrument displacement is implemented through a sequence of single linear steps with identical length and direction, called macro stimuli. The tool-tissue interactions are implemented through an end-effector at the tip of the tool. Every macro stimulus leads to micro displacements (micro stimuli) at the contact tissue point in a direction perpendicular to the contact tangential plane. The micro-stimuli interact with the tissue and lead to its reaction, which modulates the stress and forms a micro force in a direction opposite to the micro displacement. The total sum of micro forces (its component in the direction of the tool displacement) is counted by a tactile sensor. It is used in the assessment of the tissue structure. In the following text, the operation of a robotic tool with a conical shape of the end-effector will be investigated. The end effector is shaped conically with an angle of the tip $2 \alpha$. The translation of the end-effector is a result of a single macro stimulus with equal steps $L_{0}$ and a direction defined by the straight line $\mathrm{O}-\mathrm{O} 1$. After each step, the contact point of the tissue and the tool performs a micro displacement in a direction perpendicular to $\mathrm{O}-\mathrm{O} 1$ with a length $D_{l}$, which leads to a micro stimulus with a length $L_{1}$ (a micro displacement perpendicular to the tangent plane to the conical contact point). The tissue reaction is the generation of the micro force $F_{m}$. Its component in the direction of the displacement of the tool $F_{m t}$ and the similar components of the micro forces generated at the other contact points are summed up and measured by the incorporated tactile sensor. A series of measurements is performed according to a pre-set time interval [2].

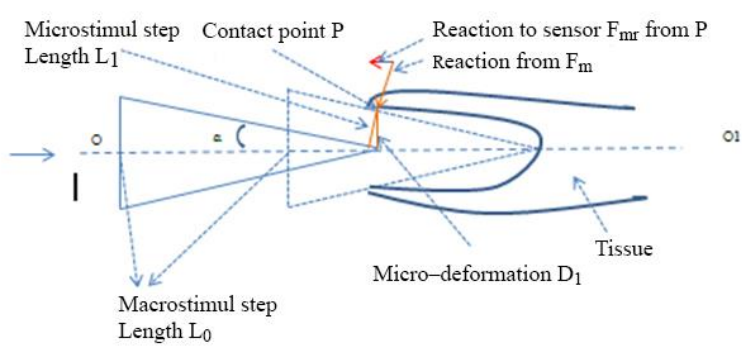

Fig. 8. Movement of the end-effector.

Fig. 8 shows the movement of the end-effector, which is done via a sequence of macro stimuli, each having a length $L_{0}$ in a predefined direction. Every macro stimulus generates micro stimuli and micro displacements with a length of $L_{1}$ at the contact points of the tissue. The following equation holds:

$$
L_{1}=L_{0} \sin \alpha
$$


The exceptions are the new contact points that occur when the tip of the tool interacts with the tissue. They are located on the surface on the cone, which is bound by its tip and its cross-section with a plane perpendicular to the axis $O-O 1$ and located at a distance $L_{0}$ from its tip.

At these points, the micro displacements are determined by:

$$
L_{1}=r
$$

where $r$ ranges from 0 to $L_{0}$

From Equation (6), it follows that:

$$
\sigma(t)=\xi \times E \times e^{-\frac{t}{\tau}}
$$

$\varepsilon$ is one monotonous function $P\left(L_{1}\right)$, whose value is constant during the test measurements of $\sigma$ :

$$
\xi=P\left(L_{1}\right)
$$

Equation (10) can be rewritten as:

$$
\sigma(t)=P\left(L_{1}\right) \times E \times e^{-\frac{t}{\tau}}
$$

The stress $\sigma(\mathrm{t})$ at the point $\mathrm{q}$ in a constant tissue area can be defined by the reaction of its deformation $L_{l}$ and it is a function of the time. If $\Delta S(q)$ is the area of a micro-surface within the end-effector surface, in which there is a contact point $q$ with stress $\sigma$, then a microforce is formed on this micro-surface, whose projection $F_{m t}(p)$ on the axis $\mathrm{O}-\mathrm{O} 1$ can be defined as:

$$
F_{m t}(p)=\sin \alpha \times \Delta S(q)
$$

Summing up $F_{m t}(p)$ at all contact points $q$, we receive the force which is measured by the tactile sensor at a given moment:

$$
F=\sum_{q \in\{\text { contact poins }\}}\left(F_{m t}(q)\right) \equiv F_{m t}(p)
$$

To refine the estimate of the magnitude of the force $F$, which is measured by the tactile sensor, let us examine in detail the force interaction between the tool and the tissue. The conical manipulator can be regarded as being composed of two segments:

1. K0, which includes the points of the conical surface that are at a distance or less than or equal to $L_{0}$ (the length of the macro-stimulation steps) from the plane, which is orthogonal to the axis $O-O 1$ and passes through its tip. $K O$ creates new contact points with the tissue and equation (8) is in force.

2. $K 1$ includes the points of the cone surface which are located at a distance larger than $L_{0}$ (the length of macro-stimulus steps) from the plane which is orthogonal to the axis $O-O 1$ and passes through its tip. $K 1$ always interacts with contact points which have already been created and for which equation (7) is in force:

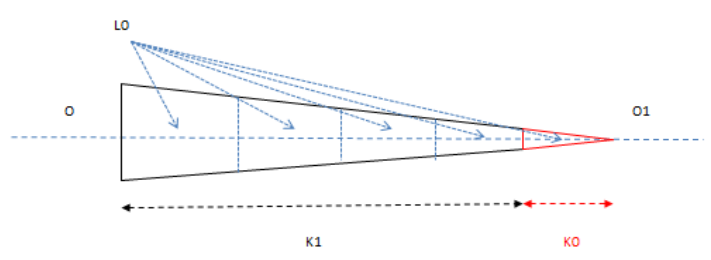

Fig. 9. Segmentation of the conical end-effector.

In Fig. 9, the segmentation of the conical endeffector is shown.

The tool performs a sequence of movements. Each one is the result of a macro-stimulus and generates translation movement with a step $L_{0}$. As a result, the segments $K 0$ and $K 1$ perform a displacement of the contact points at a distance $L_{l}$ perpendicular to the surface of the cone and determined by equation (8) or equation (7), respectively. The tissue reacts at each contact point and a micro force $F$ is formed along the axis $O-O 1$ and the total force $F$ is obtained by equation (13):

$$
F=F 0+F 1
$$

where $F 0$ is the sum of the force reactions formed at the contact points of the tissue with $K 0$ and $F 1$ is the sum of the force reactions formed at the contact points of the tissue with $K 1$.

When performing a sequence of macro stimulations, the force measured by the sensors tends to increase. This is due to the increase in the number of contact points in a series of consecutive macro stimulations:

1. For the first macro stimulus this includes only the new contact points that are formed by $\mathrm{K} 0$;

2. For the second macro stimulus, this includes the new contact points that are formed by $K O$ and the existing contact points that are formed by $K 1_{l}$;

3 . For the $n+1$ stimulus, this includes the new contact points that are formed by $K_{0}$ and the existing contact points that are formed by $K 1_{n}$;

The reaction of the tissue to every segment $K 0, K 1_{l}$, $\ldots, K 1_{n}$ is as follows generates a corresponding force that is measured by the sensor. In the general case, for predefined $L_{0}$ and $\alpha$, the tissue reaction measured by the tactile sensor for the $n^{\text {th }}$ macro stimulus can be written as follows:

$$
F n=F 0+F 1_{n}
$$

It can be shown that there is a common multiplier $e^{-\frac{t}{\tau}}$ in both addends on the right side of equation (15) because of which: 


$$
F n=e^{-\frac{t}{\tau}} \times M\left(L_{0}, E, \alpha, n\right)
$$

where $F n$ is the force measured for the $n^{\text {th }}$ macro stimulus. The function $M\left(L_{0}, E, \alpha, n\right)$ depends on the parameters of the tool, the elastic modulus of the tissue $E$, and the sequential index $n$ of the macro stimulus. A characteristic of this function is that it does not change its value during the time necessary to perform the macro stimulus. Due to this reason, the force $F n$ can be measured at the beginning of the second phase of the macro stimulus (for $t=0$ ). Subsequently, the value of $\tau$ can be determined through a series of measurements.

\section{A device for micro and macro stimulation}

Every macro stimulus includes two operational phases $[3,4]$ :

1. Generation of stimulation. The tool performs a translation step that moves the end-effector. During the execution of this phase, a sufficient force is applied to the tool to ensure the movement and the deformation of the tissue under test.

2. Holding phase. It starts after the successful completion of the first phase. During this phase, a retention force is applied to the manipulator, which is less than the displacement force but sufficiently large to ensure that the tissue deformation that occurred during the first phase is retained. A series of successive measurements of the force generated by the tissue reaction are done over time by means of a tactile sensor built into the slider of the manipulator.

Fig. 10 shows a time diagram representing the execution of two macro stimuli along with the device control and measurement values.

From Fig. 10, it can be concluded that a good technical solution of the macro stimulator generator is the use of a linear actuator based on a stepper motor and controlled by a specialized driver under the control of a microcontroller. Fig. 11 shows the construction of such a device together with the mounted conical manipulator.

The linear actuator executes a translation movement of the manipulator by performing a step of pre-defined length $L_{0}$. To ensure that the movement is executed, an incremental encoder is coupled to the bipolar stepper motor. Each step is initiated by the microcontroller, which sends a narrow pulse to the motor driver and processes the encoder data to monitor the completion of the step. Then, it starts the second macro stimulation phase. During the second phase, the microcontroller performs successive measurements of the built-in tactile sensor readings. During this period, the bipolar stepper motor is in retention mode, which is ensured by maintaining a constant current through its windings without initiating a new step (the driver always works in active state). The holding phase ensures the correct measurement of the tactile sensor response force and the processing of this data by the micro controller.

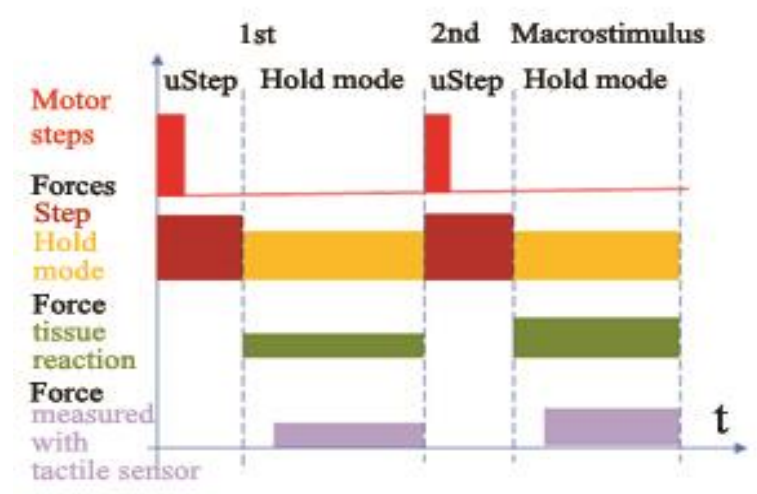

Fig. 10. Time diagram of a macro stimulus.

The macro stimulus generator must have specific electromechanical properties:

1. It must be capable of generating steps of a very small length $L_{0}$. This is necessary in order to emulate the requirements of the Time and Relaxation test as well as possible. The test assumes an instantaneous generation of the deformations in the examined tissue and a minimal impact on the tissue by the manipulator;

2. It must block the movement of the manipulator during the second phase. This is related to the construction of the device and the manipulator body, which houses the tactile sensor that measures the force of the tissue reaction;

3. In the course of successive macro stimuli, the step that corresponds to the last macro stimulus must begin from the position at which the previous macro stimulus has been completed.

The device shown in Fig. 11 (a linear actuator built on the basis of a bipolar stepper motor with a built-in encoder and a specialized driver under the control of a microcontroller) fulfills all these requirements and provides the generation of steps in the range of 8 to 48 micrometers.

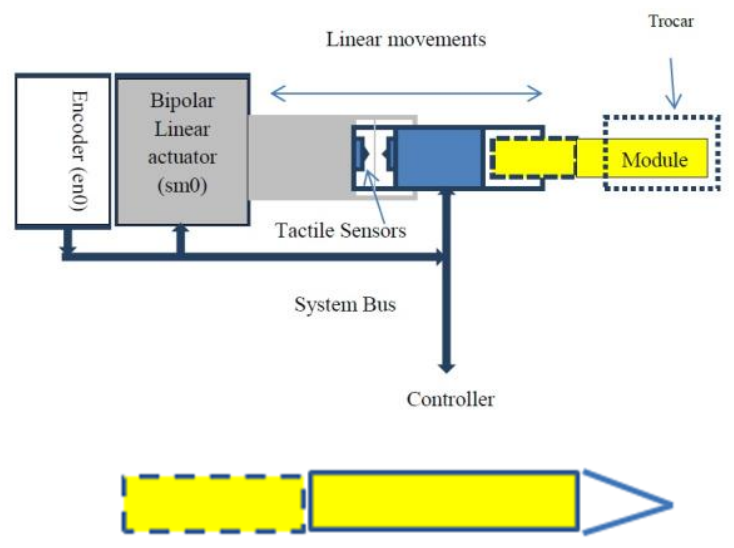

Fig. 11. A linear actuator based on a bipolar stepper motor with a conical manipulator and a built-in tactile sensor. 


\section{Analysis of the output signal from the research instrument.}

The purpose of the research instrument is to form a series of macro stimuli to create deformations in the contact points with the tissues and to analyze the incoming information about the force of the reaction from the built-in tenzo-sensor. On the basis of this analysis, the relaxation time $\tau$ is determined, which characterizes the mechanical properties and the structure of the tissue [3].

The analysis is done separately for each of the generated macro stimuli. It involves the formation of a manipulator translation movement with a step $L_{0}$ (the first phase of the macro stimulation). Immediately after the motion, the initial reaction force $F_{b}$ is read out by means of the tactile sensor. At this point, a timer is activated, which measures the time intervals at which the reaction force is measured during the second phase of the macro stimulation. During these measurements, the condition for the newly measured force $F_{c}$ to have a magnitude less than or equal to $F_{b} / e$ is monitored, where " $e$ " is the Euler number. When this condition is met, it is assumed that the readings of the timer are equal to the relaxation time $\tau$. The second phase of the macro stimulus ends and it is possible to start the next macro stimulus. Typically, macro stimulations are generated at fixed time intervals, e.g. $100 \mathrm{~ms}$. This value is also used as a time-out when determining $\tau$. The actions described above are illustrated in Fig. 12.

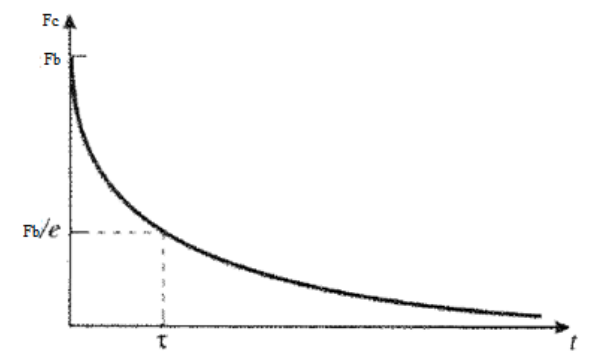

Fig. 12. Determination of the "relaxation time $\tau$ " parameter.

By performing a sequence of macro stimulations and determining $\tau$ for each of them, we can scan the structure of the biological tissue. The inability to measure $\tau$ within the limiting period or a sharp change in the value of this parameter are indications of abnormal areas in the tissue that may be the result of the presence of tumor nodes in its composition.

\section{Discussions}

The method for analysis of the structure of biological tissues presented in this paper has a number of advantages:

1. The analysis is made on the basis of one microparameter $\tau$ that determines the type of viscoelastic environment. This parameter is measured by means of macro values - force and time - separately for each macro stimulus. There is no need to measure the deformation and strain, which are often inaccessible to the researcher;

2. $\tau$ is the result of direct force measurements, which integrate the micro responses at every contact point for the given macro stimulus. In this context, the parameter filters the noise and reports an average relaxation time;

3. This method is useful for real-time diagnostics and force feedback interaction in surgical robotics. It is possible to scan accurately the change of $\tau$ and locate precisely the areas where the parameter makes sharp changes in its value.

\section{Conclusions}

In laparoscopic surgery, it is very important to provide adequate information to the surgeon about the contact points of the instrument with the tissue. This information has to be received in real-time and synchronously with the control of the movements of the devices and it has to be the basis for the acceptance of different solutions by the control device. The macro- and micro-stimulation method presented in the paper is applied for diagnostic purposes and detects changes in the tissue structure. It allows the real-time monitoring of the relaxation time parameter, which gives information about the structure and mechanical properties of the biological tissue. The use of macro-stimulations to control not only mechanical but also other physical processes will create opportunities for the realization of new technical solutions.

This paper is supported by the National Scientific Program "Information and Communication Technologies for a Single Digital Market in Science, Education and Security (ICTinSES)", financed by the Ministry of Education and Science.

\section{References}

1. N. O'Zkaya, Margareta Nordin, David Goldsheyder, Dawn Leger, Fundamentals of Biomechanics: Equilibrium, Motion, and Deformation, ISBN 978-14614-1149-9, E-ISBN 978-1-4614-1150-5, Springer, DOI 10.1007/978-1-4614-1150-5, pp. 1- 286, (2012)

2. V. Ivanova., D. Bachvarov., A.Boneva., An advanced robot system for diagnostic and therapeutics tasks with application in Laparoscopic surgery., Journal of Computer Engineering and information technology ., Volume 7, Issue 12018 DOI 10.4172/2324-9307.1000202, ISSN: 24128856 (Online), , SciTechnol, pp. 1-9, (2018)

3. G. Dagnini, Laparoscopy and Imaging Techniques, ISBN-13: 978-3-642-74647-5, E-ISBN-13: 978-3642-74645-1, Springer, DOI:10.1007/978-3-64274645-1, pp. 1- 205, (2012).

4. D. Knudson Fundamentals of Biomechanics, ISBN 978-0-387-49311-4, E-ISBN 978-0-387-49312-1, Springer, pp. 1- 250, (2007). 